\title{
6.02 \\ Consumo seguro e saúde: desafio e articulação, também no campo do Direito Sanitário
}

Safety consumer and health: challenge and articulation, also in the field of health law

\section{Neilton Araujo de Oliveira}

Médico, Especialista em Saúde Pública e em Políticas e Estratégias Nacionais, Mestre em Saúde Coletiva e Doutor em Ciências, Professor da Universidade Federal do Tocantins (UFT) e Diretor Adjunto da Agência Nacional de Vigilância Sanitária (ANVISA). Brasília, Brasil.

Resumo: A saúde, decorrente de um conjunto de fatores sociais, políticos, econômicos, ambientais e culturais, para ser efetivada necessita de variadas abordagens. Na esteira das discussões da conferência mundial de determinantes sociais da saúde, 'consumo' se apresenta como importante determinante da saúde exigindo articulação/interação multiinstitucional e intersetorial, referenciada na discussão da integralidade da saúde. Este trabalho discute a relação 'consumo seguro/saúde' e apresenta resultados de uma oportuna articulação que Anvisa, Inmetro e Senacon vêm construindo, em sintonia com o movimento da Rede Consumo Seguro e Saúde das Américas (RCSS), na perspectiva de conferir maior proteção sanitária aos consumidores. Divulgar e ampliar a discussão sobre o tema é objetivo deste estudo descritivo/reflexivo, realizado com levantamento de documentos, observação participante e sistematização de propostas sobre a temática e estratégias voltadas ao fortalecimento desta iniciativa brasileira. Um aspecto fundamental nesse contexto é a noção de risco e responsabilidade, especialmente quando se trata de acidentes de consumo. Se uma criança engasga e se asfixia com uma peça pequena de brinquedo que se solta, ou se estrangula numa cordinha de sua roupa, a noção ainda preponderante é que se trata de um descuido dos pais e, portanto, deixa de se reconhecer aí um acidente de consumo. Nos países desenvolvidos, "acidentes de consumo" constituem preocupação prioritária, enfrentada, ora pelo setor sanitário, ora pela justiça, mas incluindo setores como educação, indústria e comércio, meio ambiente, dentre outros. Para o Brasil, esta temática se coloca como urgente desafio a ser enfrentado, também no campo do Direito Sanitário.

Palavras-chave: Consumo seguro; consumidor; articulação em rede; Direito Sanitário.

Key words: Consumer Safety; consumer; coordination network; Health Law. 


\section{Introdução}

Dentre as muitas abordagens e outras diferentes reflexões sobre a saúde no contexto atual, a dimensão individual e centrada/referenciada no modelo biologicista, identificada muito mais com a doença, ou seja, a falta da saúde, ainda sobrepõe outras concepções, com uma consequente e forte dificuldade de se reconhecer a saúde na sua verdadeira natureza, a da integralidade e da sua multideterminação (OMS, 2011), concepção esta que, por outro lado, vem sendo gradativamente apresentada e defendida por mais pessoas, muitos estudos e em diferentes espaços, embora muito ainda na contra hegemonia, porém tendo como linha referencial a discussão da integralidade em toda sua abrangência e amplitude: do individual ao coletivo, do local ao global.

Assim, a saúde, direito humano essencial, é cada vez mais compreendida como socialmente multideterminada - decorrente de um conjunto de fatores sociais, políticos, econômicos, ambientais e culturais - e, desde a CF/1988 (Brasil, 1988), estabelecida como um direito de cidadania que, no entanto, para ser efetivado, necessita de variadas estratégias, no que tem resultado uma sólida tensão paradigmática, tanto na dimensão dos conceitos, quanto na dimensão da compreensão e defesa dos modelos de atenção à saúde (Oliveira, 2012).

Nesta direção, à luz e na esteira das discussões da Conferência Mundial de Determinantes Sociais da Saúde (CMDS, 2011), recentemente realizada no Brasil e discutindo inúmeros temas e com ampla participação de países de todos os continentes, um novo tema - Consumo Seguro - começa a ser visto e discutido para além da dimensão econômica, ao se apresentar como um importante determinante social da saúde que, para seu enfrentamento, exige uma articulação e interação multiinstitucional e intersetorial. Para isso, a disponibilidade de informações rápidas e claras, cotidianamente disponível para as autoridades, bem como para toda a população, caracteriza-se com uma necessária ferramenta de proteção sanitária de todas as populações, em todas as partes do planeta, ricas e pobres, desenvolvidas ou não, mas especialmente as mais vulneráveis socialmente. Isso tem obrigado os sistemas nacionais a desenvolverem uma capacidade de forte e permanente vigilância à saúde, também, a produtos e serviços de consumo inseguros.

No Brasil, mais recentemente, esse assunto vem provocando uma crescente mobilização e constituindo um movimento plural, adquirindo alguma visibilidade e 
organicidade, inclusive nos âmbitos regional e local (O Globo, 2013) e envolvendo diferente sujeitos sociais, acadêmicos e institucionais, especialmente organismos ligados aos setores de defesa e proteção do consumidor e da proteção e vigilância da saúde.

Portanto, no campo do Direito Sanitário, não é por outra razão que essa discussão vem, também, adquirindo corpo e constituindo um desafio novo e mobilizador, para diferentes agentes, e propiciando uma oportuna articulação interinsticucional.

É com esse sentido que o presente trabalho foi realizado, buscando contextualizar essa temática no mundo e no Brasil, relatar os principais passos já percorridos dessa trajetória, com a pretensão de discutir a relação entre Consumo e Saúde, no contexto da integralidade da Saúde, e apresentar os resultados já produzidos pelo movimento do que se denominou "Grupo Consumo Seguro e Saúde Brasil - RCSS-GT.Brasil” que, atualmente, é integrado pela Agência Nacional de Vigilância Sanitária (ANVISA), Instituto Nacional de Metrologia, Qualidade e Tecnologia (INMETRO), Secretaria Nacional do Consumidor (SENACON) e Ministério da Saúde/Secretaria Nacional de Vigilância em Saúde (MS-SVS) e os respectivos Sistemas Nacionais de Vigilância Sanitária, de Metrologia e de Proteçao do Consumidor.

Portanto, em sintonia com o esforço interamericano de discussão e organização da "Rede Consumo Seguro e Saúde das Américas-RCSS" (OEA, 2010), na perspectiva de conferir maior proteção sanitária aos consumidores, o tema carece de maior maior difusão e debate. Então, a oportunidade da realização conjunta dos Congressos (III Iberoamericano e II Brasileiro) de Direito Sanitário, no Brasil, em outubro/2013, configura-se como momento e espaço propícios para ampliar o debate desse complexo desafio, na expectativa de , assim, contribuir para que essa articulação Consumo Seguro e Saúde seja considerada e assumida como própria, também, do campo do Direito Sanitário (Oliveira, 2013).

\section{Metodologia}

Trata-se de um estudo descritivo/reflexivo, realizado com levantamento de bibliografia e documentos, observação participante e sistematização de propostas atualmente em discussão e/ou formulação sobre o tema no país e diferentes nações 
do mundo, com a definição de estratégias e proposições voltadas para o fortalecimento desta articulação e iniciativa, no Brasil e nas Américas.

\section{Resultados e discussão}

\section{A noção de risco e de quem é responsável pela segurança de produtos de consumo.}

Dentre os principais pontos de destaques abordados como resultados desse estudo, e uma constatação muito oportuna à presente discussão, que constitui um aspecto fundamental no presente contexto, é a própria noção de risco e de responsabilidade, relacionadas à segurança de consumo, especialmente quando se trata de acidentes de consumo.

Enquanto nos paises desenvolvidos essa questão já esteja devidamente bem colocada e bastante equacionada - valorizando-se sobremaneira a segurança de produtos de consumo e localizando no respectivo fabricante do produto a tarefa e a responsabilidade pela segurança conferida a seus produtos -, no Brasil e nos países da América Latina, por outro lado, o risco coloca-se mais como desdobramento e consequência de um descuido do usuário e a responsabilidade pela segurança dos produtos é muito mais vista e localizada em quem usa o produto, ou ainda como sendo uma deficiência/ descuido, culpa mesmo dos órgãos governamentais que os regulam.

Assim, se por exemplo uma criança se engasga e se asfixia com uma peça pequena de brinquedo que se solta, ou ainda se estrangula numa cordinha de uma persiana, ou de uma roupinha que veste, a noção ainda preponderante, no Brasil e na América Latina, é a de que se trata de um descuido dos pais e cuidadores e, portanto, deixa de se reconhecer a insegurança do produto e, por consequência, não se reconhece aí um acidente de consumo.

Desta forma, observa-se que nos Estados Unidos da América, União Europeia, Canadá, Japão, Austrália e na grande maioria dos paíse desenvolvidos, "acidentes de consumo" constituem uma preocupação importante compartilhada, por um lado, conferindo aos fabricantes a responsabilidade pela segurança e correta informação/orientação de uso do seu produto; por outro lado, conferindo aos órgãos reguladores a vigilância/monitoramento da segurança de produtos e a correspondente 
penalização (exemplar, por sinal) do fabricante, em caso de inobservância ou descumprimento, tanto intencional quanto involuntário, da sua responsabilidade de conferir segurança ao seu produto. Isso é regra geral exigida pelos consumidores e um processo do qual diferentes setores participam e se articulam, ora liderados pelo setor sanitário, ora por órgãos da ustiça, e que inclui obrigatoriamente outros setores como educação, desenvolvimento, indústria e comércio, meio ambiente, dentre outros.

Para o Brasil, que nas últimas décadas vem alcançando patamares significativamente mais elevados em termos de crescimento econômico, e de desenvolvimento social, político, sanitário, educacional etc, esta temática (importante também no campo de direitos do consumidor) se coloca como um grande e urgente desafio a ser enfrentado. Para isso, é fundamental difundir e discutir os espaços trabalhados e estratégias já formuladas/implantadas na busca crescente de mobilizações insterinstitucionais e intersetoriais para estabelecer um confiável sistema de comunciaçao, com oportunos "alertas rápidos" sobre (in)segurança de produtos de consumo. Assim, oportunas informações e novos conhecimentos certamente contribuirão para a adequada compreensão de risco e a necessária e correta tomada de decisão, cuidando para envolver todos os diferentes atores participantes nesse complexo processo... e, como dizia Hartz (2001), "para problemas complexos, soluções complexas!".

\section{A trajetória complexa - ainda recente, mas já siginificativamente exitosa - do movimento "em rede" Consumo Seguro e Saúde, no Brasil e nas Américas.}

Sem pretender aqui fazer apenas um 'relatório', muito menos querendo ter a pretensão de apresentar uma descrição completa, embora fiel, de todos os procedimentos que compõem essa já sólida articulação brasileira e interamericana, "em rede", sobre a temática "consumo seguro e saúde", porém com a expectativa de trazer nesse momento informações preciosas de tal inciativa, que possam contribuir para a motivação, mobilização e engajamento de pessoas e instituições nesse esforço multinacional e intersetorial, o principal propósito dos tópicos descritos e discutidos a seguir, concentra-se na busca de trazer para o campo de direito sanitário a ampliação e aprofundamento do debate sobre segurança de produtos e proteção sanitária dos consumidores. 
A Rede Consumo Seguro e Saúde das Américas (RCSS) é um primeiro esforço interamericano para a conformação e consolidação dos sistemas nacionais e regionais de vigilância de mercado, destinados a promover a segurança de produtos (OEA, 2010). Fundamentalmente, uma estratégia a serviço das autoridades e dos consumidores das Américas, com ações em três eixos ou focos principais:

a) Intercâmbio de informações e troca de experiências, difusão da temática proteção sanitária do consumidor e educação sobre segurança dos produtos de consumo e seu impacto na saúde;

b) Formação/capacitação de técnicos e autoridades das Américas, com vistas a desenvolverem capacidades específicas sobre sistemas de gestão de vigilância de mercado e segurança de produtos;

c) Desenvolvimento de um portal na internet (www.oas.org/rcss), já em funcionamento, que proporciona fácil acesso a informações relevantes sobre produtos considerados inseguros, por mercados de todo o mundo, com avançados sistemas de alertas.

A RCSS, fruto da cooperação de diversas instituições e países, coordenada por uma parceria entre a Organização dos Estados Americanos (OEA) e Organização Pan-Americana da Saúde (OPAS), apoiada por um "Grupo Técnico Assessor-GTA", integrado por representantes de diversos países (incluindo o Brasil, com um papel protagonista no processo), conta também com a participação da sociedade civil representada por Consumers International. Esforço esse em sintonia e em atendimento ao mandato outorgado pela Carta da OEA quando estabelece que os Estados Membros devem realizar esforços com o fim de prover bens e serviços adequados e seguros para os seus consumidores.

O objetivo maior da RCSS é contribuir para a conformação e consolidação dos sistemas nacionais e regionais destinados a fortalecer a segurança do consumo, mediante a detecção rápida e a ação coordenada, com vistas a evitar ingressos de produtos inseguros nos mercados americanos e, assim, proteger a saúde dos consumidores (OEA, 2010).

No caso específico dos consumidores, considera-se imprescindível uma vigilância sobre produtos e serviços em todos os países do planeta, o que tem produzido acentuadas assimetrias na proteção à saúde das populações consumidoras, na medida em que muitos países ainda não contam com recursos 
necessários para isso, não dominam os novos conhecimentos e, até mesmo, não dispõem de legislação específica para assegurar efetiva segurança sanitária no consumo de produtos.

Assim, mais do que oportuna, pode ser considerada necessária e muito estratégica uma articulação nos âmbitos mundial, regional e nacional para dotar cada país com condições de desenvolver sua capacidade sobre uma competente vigilância de segurança de produtos de consumo.

Em síntese, foi com este sentido e fundamentado nessas referências que OEA e OPAS, em meados de 2009 realizaram uma primeira reunião/consulta técnica, em Washington, promovendo um primeiro levantamento de informaçoes sobre este assunto e com vistas a inciar uma articulaçao entre os países das Américas. Uma segunda consulta técnica, com especialists nessa temática, foi realizada no mesmo ano, também em Washington, quando os representantes do Brasil (ANVISA e DPDC), presentes desde a primeira reunião, assumiram papel de destaque, e protagonista na proposição e condução de encaminhamentos visando a formulação e criação da RCSS.

A esta altura, DPDC e ANVISA contavam com um Termo de Cooperação, previamente estabelecido para no Brasil atuarem com esforços conjuntos na proposição de medidas relativas à proteção da saúde dos consumidores, incialmente pensado em algo como que um "observatório" e, logo depois, numa "articulaçao em rede" (Oliveira, 2010). Muito em função da compreensão de que saúde do consumidor, na perspectiva de direito fundamental - propugnado na CF/1988, na Lei 8.078/90 (Código de Defesa e Proteção do Consumidor), Leis 8.080/90 e 8.142/90 (Leis Orgânicas da Saúde e do SUS) -, exige a articulação integrada e eficiente na provisão dos serviços e de produtos indispensáveis à garantia do bem-estar das pessoas, seguramente trata-se de uma tarefa de fôlego que busca, inclusive, maior eficácia na formulação de Políticas Públicas e Sistemas de Vigilância, para fortalecimento da proteção sanitária do consumidor.

Em parte, isso explica a articulada e forte participação do Brasil, logo no início do movimento de formulação e criação da RCSS, apoiando e mobilizando autoridades e técnciso dos demais países das três Américas e, especialmente do Brasil. Isso foi fundamental para a propsiçao e aprovação, na Assembleia Geral de OEA, da Resolução AG/RES. 2494 XXXIX-0/09, que explicitava o compromisso com 
a "Proteção do Consumidor" e solicitava à Secretaria Geral de OEA o reforço dos mecanismos de cooperação existentes entre os países membros.

Em seguida, a ANVISA aportou via Termo de Coperação (TC 41, MS-OPAS) um primeiro recurso financeiro, num total de $U \$ 110,000.00$, que possibilitou apoiar os primeiros movimentos de implantação da RCSS:

- $\quad$ Seminário sobre Vigilância de Mercado, realizado em Lima/Peru, em agosto/2010, quando foi apresentada e aprimorada a proposta da "articulação em rede", que se propunha, e foi discutido um protótipo do Portal de Alerta Rápidos da RCSS;

- Reunião do Grupo Técnico Assessor da RCSS (GTA-RCSS), agosto/2010, em Lima/Peru;

- Desenvolvimento do projeto Portal de Alertas Rápidos, coordenado e sediado no site de OEA, cujo lançamento posteriormente seria feito no Conselho Permanente de OEA;

- $\quad 1^{\circ}$ Curso "Sistemas de Vigilância de Mercado e Segurança de Produtos" OEA/OPAS, on-line (quatro semanas) e presencial (uma semana), em cooperação com Universidade Pompeu Fabra e Agência Catalã de Segurança de Produtos, ambas de Barcelona-Espanha, e com uma presença majoritária de autoridades de defesa do consumidor, o que fez com que ANVSA passasse a atuar mais fortemente junto à OPAS para mobilizar autoridades sanitárias de outros países nessa articulação.

Internamente na ANVISA, crescia o interesse nesse tema e a motivação de servidores, culminando com a criação em fevereiro de 2011 do Grpo Consumo Seguro Anvisa (RCSS-GT.Anvisa), e a puplicação da respectiva portaria, oficializando a iniciativa.

$\mathrm{Na}$ sequência, a cooperação nacional ANVISA-DPDC, ampliada com a integração à mesma, pelo INMETRO, aportou mediante um TC ANVISA-OEA, em novembro/2010 o montante de U\$255,241.07 que entre outras atividades permitiram concretizar:

- Implementação da RCSS e "Portal de Alertas Rápidos" e consultorias;

- Duas novas reuniões do Grupo Técnico Assessor (GTA-RCSS);

- $\quad 2^{\circ}$ Curso on line e presencial "Sistemas de Vigilância de Mercado sobre Segurança de Produtos de Consumo" (junho/ 2012) - ainda em cooperação com 
Universidade Pompeu Fabra e apoio da Agência Catalã de Consumo, Monitoramento de Mercado e Segurança de Produto, de Barcelona.

- $\quad$ "Seminário Internacional sobre Consumo Seguro e Reparação de Danos causados por Produtos Defeituosos" - novembro, 2011, em Bogotá-Colômbia;

- $\quad$ Produção e divulgação de material Consumo Seguro e Saúde e sobre a RCSS e o Portal de Alertas Rápidos.

O Portal de Alertas Rápidos, cujo lançamento foi feito por representantes do Brasil e EUA, em novembro 2011, no Conselho Permanente de OEA (foto abaixo), está disponível no site da OEA ( $\underline{\text { www.oas.org/rcss) }}$ ) em quatro idiomas (espanhol, português, francês e inglês) e reúne Alertas Rápidos sobre insegurança de produtos de todo o mundo, na língua original em que os alertas são publicados.

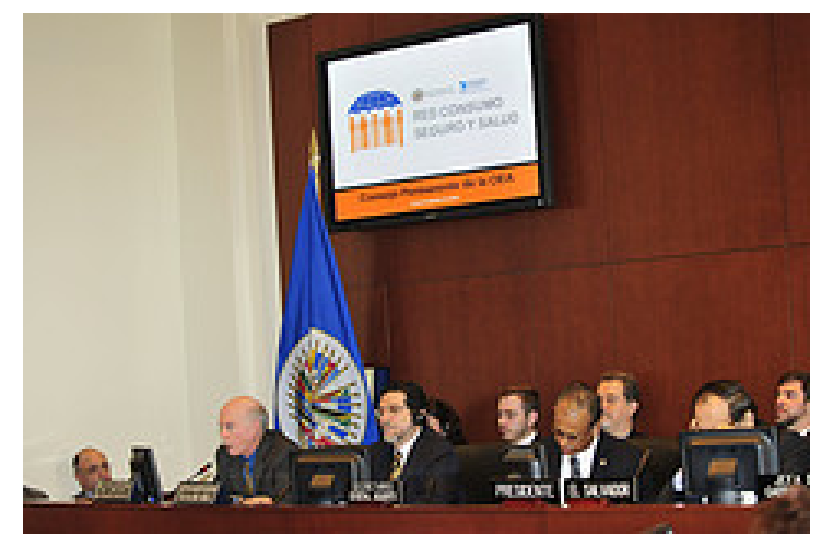

Figura 1 - Cerimônia de lançamento do Portal de Alertas Rápidos, no Conselho Permanente de OEA. Novembro/2011.

Cerca de um ano depois, em junho/2012, novamente liderado pelo Brasil, mas com atuação forte e importante de vários outros países das Américas foi aprovada na Assembléia Gera; de OEA uma nova proposição, pela Resolução AG/RES.2712 (XLII-0/12) colocando para a Rede Consumo Seguro e Saúde das Américas (RCSS) uma nova e fundamental etapa: desenvolver e implantar o Sistema Interamericano de Alertas Rápidos (SIAR)-Segurança de Consumo. A mesma resolução aprovou a criação do Fundo Voluntário da RCSS e solicitava, a todos os países membros, esforços de seus governos para contribuírem.

Nesse mesmo período, tendo sido criada anteriormente a Secretaria Nacional do Consumidor (SENACON/MJ), no Brasil era oficializado por portaria conjunta dos 
três órgãos o Grupo Consumo Seguro e Saúde Brasil (RCSS-GT.Brasil) integrado por ANVISA, INMETRO e SENACON, o que favoreceu a intensificação de antigas e novas atividades relacionadas a consumo seguro e saúde, dentre elas:

$>\quad$ Intensa mobilização e articulação institucional e de pessoas;

$>\quad$ Lançamento do Sistema Nacional de Alertas - Recall SENACON;

> Audiência pública "Consumo Seguro e Saúde" na Comissão de Direito Humanos do Senado Federal (CDH/SF), com cobertura e veiculação pela TV Senado, como primeira atividade da $1^{a}$ Semana da Vigilância Sanitária no Congresso Nacional, promovida pela ANVISA, em maio de 2012 (Anvisa, 2012);

$>\quad 1^{\circ}$ Seminário Internacional Consumo Seguro e Saúde promovio pelo RCSS-GT.Brasil em junho/ 2012, em Brasília (Anvisa, 2012);

$>1^{\mathrm{a}}$ Oficina Temática sobre Saúde e Segurança, promovida ela SENACON em agosto 2012, em Brasília;

> Audiência pública "Consumo Seguro Saúde", conjunta na CDH/SF e CAS/SF, também veiculada pela TV Senado, dentro da $2^{a}$ Semana da Vigilância Sanitária no Congresso Nacional, promovida pela ANVISA, em maio de 2013, em Brasília (OEA, 2013).

"I Semana Nacional Consumo Seguro e Saúde" (Anvisa, 2013), promovida pelo RCSS-GT.Brasil, em junho/2013, com um conjunto amplo de atividades, dentre elas o II Seminário Internacional Consumo Seguro e Saúde (Anvisa, 2013 e IDEC, 2013).

Reforçando a articulação continental, nos primeiros meses de 2013 ocorreu a $3^{a}$ edição do curso sobre vigilância de mercado e segurança de produtos, desta feita com uma presença maciça de autoridades sanitárias de diveros países das Américas, e está em fase de sistematização os dados levantados e informados pela quase totalidade dos países das três Américas, sobre normas e procedimentos regulatórios, para compor uma coletânea a ser publicada como "Atlas Consumo Seguro e Saúde das Américas".

Uma participação ativa e apoio importante, nas atividades da RCSS, tem sido feito por Consumer Product Safety Commission-CPSC, dos EUA (http://www.cpsc.gov/), inclusive com suporte especial por ocasião da semana presencial, em Washington, nas três edições do curso Gestão de Sistemas de Vigilância de Mercado e Segurança de Produtos. 
Internacionalmente diversas articulações e atividades vêm sendo desenvolvidas e, em algumas delas, representantes de diversos países, incluindo o Brasil, vêm participando nos últimos anos, apresentando os avanços da RCSS e discutindo segurança de produtos e saúde dos consumidores, e suas relações intercontinentais, tais como alguns simpósios internacionais: Organisation for Economic Co-operation and Development (OCDE, 2012) e The International Consumer Product Health and Safety Organization (ICPHSO, 2013).

\section{Sistema de Informações de Acidentes de Consumo - SIAC}

Diante da complexidade do tema Consumo Seguro e Saúde, diversas tarefas se colocam como importantes e prioritárias para serem enfrentadas. Dentre estas se destacam, por exemplo, mapear todos os problemas, identificar temas comuns e definir conceitos e realizar projetos-pilotos relacionados a informações sobre acidentes de consumo.

Esta é uma iniciativa fundamental, e muito importante no contexto da segurança de produtos de consumo e saúde dos consumidores, que em diversos países desenvolvidos é uma realidade, há décadas. No Brasil, sabe-se que os diversos órgãos e instituições possuem diferentes bancos de dados, porém estes não se "falam", não abrangem todas as dimensões do problema e nem informam a real situação da questão. Ou seja, precisamos qualificar esses dados e sistematizar a informação para estar disponível, de forma precisa e completa. Isso depende da integração dos atores do processo e de acesso fácil e pronto para a tomada de decisão, no sentido de reduzir e/ou prevenir os diferentes tipos de Acidentes de Consumo.

O DPDC/SENACON, a alguns anos, já vem mobilizando a participação de diversas instituições em um "Grupo de Estudos Permanentes de Acidentes de Consumo (Gepac)", que possibilitou diferentes ações e alguns recaal de produtos, para proteger os consumidores (MJ, 2013). O INMETRO, por sua vez, há seis ou sete anos, conta com um Sistema de Monitoramento de Acidentes de Consumo (Sinmac), cuja 'notificaçao' é feita pelos próprios consumidores (Inmetro, 2013).

Mais recentemente, visualiza-se procedimentos bastante concretos na direção de o país contar com um efetivo e eficiente Sistema de Informações de Acidentes de 
Consumo (SIAC). Nos últimos meses, a sua criação vem sendo discutida no âmbito do RCSS-GT.Brasil, que foi ampliado em junho/2013 com a participação da Secretaria Nacional de Vigilância em Saúde do Ministério da Saúde (SVS/MJ). Na primeira etapa, numa cooperação entre SVS/MS, ANVISA e SENACON, serão notificados compulsoriamente - com o preenchimento de uma ficha on line (anexo 1), pelos serviços de urgência/emergência e hospitais do país -, somente os "acidentes graves", cujas informações servirão para que os órgãos correspondentes adotem as medidas de correção/prevenção relacionadas a cada produto, ou serviço, que originou o acidente de consumo.

Ademais, já estão elencadas outras operações estratégicas com vistas ao acompanhamento e condução do processo, que é complexo, e sua ampliação e aperfeiçoamento de forma permanente, sabendo-se que muitas dificuldades só aparecerão no decurso do projeto. E, também, é preciso considerar como determinante para sua consecução a divulgação de resultados para a sociedade.

\section{Algumas diretrizes e outros desafios da articulação 'em rede' Consumo Seguro e Saúde}

O conjunto de atividades e acontecimentos no âmbito da RCSS é muito amplo e diverso, com relatos e notícias sobre uma boa parte deles na página da ANVISA, INMETRO, RCSS e SENACON, estando já consolidadas diversas diretrizes e muitos resultados, porém ainda são diversos os desafios que se colocam como prioritários no momento, tais como:

- Estabelecimento de linguagem comum sobre segurança de consumo;

- Estímulo à formação de Grupos de Trabalho específicos;

- Harmonização das iniciativas regionais e sub-regionais;

- Promoção de acordos de cooperação em âmbito subregional e regional;

- Interlocução e articulação internacionais - OCDE, RAPEX, ICPHSO etc;

- Desenvolver capacidades nacionais no campo de Consumo Seguro e Saúde;

- Fortalecimento dos sistemas nacionais com enfoques institucionais e multidisciplinar;

- Implementar Programas e Políticas Públicas relacionadas a consumo seguro e saúde;

- Intensificar e ampliar nos demais países das Américas o debate atual, intenso e articulado sobre segurança de produtos e proteção sanitária dos consumidores, a 
exemplo do que tem conseguido Brasil, Chile, Peru, Colômbia, Costa Rica, EUA, República Dominicana, Suriname, México, dentre outros);

- Construir estratégias para a incorporação da Sociedade Civil;

- Consolidar a articulação de esforços interinstitucionais e intersetoriais sobre o tema;

- Aportar novos recursos financeiros, via fundo voluntário (RCSS-OEA) face às necessidades de melhorias estruturais da RCSS, de estabelecimento de fluxos adequados de trabalho e de harmonização entre conceitos e práticas.

- Implantar e consolidar o Sistema Interamericano de Alertas Rápidos - SIAR.

Para fazer frente a estes e outros desafios no contexto da RCSS, neste momento, em nome do RCSS-GT.Brasil, a ANVISA estabeleceu um novo Termo de Cooperação com a OPAS, no total de U\$ 235,470.00 e, também em nome do RCSSGT.Brasil, INMETRO realizou um aporte à OEA de US 217,000.00 que, juntos, possibilitaram/possibilitarão em 2013 e 2014:

- $\quad 3^{a}$ edição do Curso "Gestão de Sistema de Vigilância de Mercado sobre Segurança de Produtos de Consumo" (já realizado em fevereiro 2013)

- Quatro Reuniões semestrais do GTA-RCSS.

- Seminário Continental Consumo Seguro e Saúde - RCSS: futuros passos, em novembro/2013.

- $\quad$ Consultorias específicas, especialmente relacionadas ao SIAR

- $\quad$ Produção e divulgação de material Consumo Seguro e Saúde e sobre a RCSS;

- Curso Consumo Seguro e Saúde Brasil - Sistema de Vigilância de Mercado e Segurança de Produtos;

- $\quad$ Seminário tripartite da RCSS - novembro 2013, Lima-Peru;

- Implantação do Sistema de Alertas Rápidos das Américas-SIAR

- Curso de Pós-graduação Gestão de Sistema de Vigilância de Mercado a definir

- $\quad$ Plano de ação e futuros passos, a ser sistematizado em novembro/ 2013.

\section{Conclusões e considerações finais}


Com o avanço da tecnologia e da globalização, antigos e novos riscos sanitários tornaram-se ameaças constantes em todas as partes do planeta, e afetando indiscriminadamente todas as populações, ricas e pobres, desenvolvidas ou não, mas especialmente as mais vulneráveis socialmente. Isso tem obrigado os sistemas nacionais a desenvolverem uma capacidade forte e permanente de vigilância à saúde, na linha da integralidade da saúde e de equilíbrio entre os interesses econômicos e sociais. Daí, a importância de mobilizar diferentes campos, inclusive o do Direito Sanitário.

A articulação Consumo Seguro e Saúde é uma atividade de características inovadoras muito claras, por natureza plural, e como tal precisa ser conduzida e desenvolvida numa percepção coletiva e participativa, buscando ampliar a atuação de outros setores e, especialmente, articular-se e integrar-se com outras iniciativas, o que só será adequadamente feito com um amplo arco de instituições e pessoas participantes e comprometidas com tal propósito.

Com a promulgação da CF/1988, dois setores vitais para emancipação cidadã da população brasileira obtiveram lugar de destaque no dia a dia das pessoas: Direito à Saúde e Proteção do Consumidor. Algumas ações de relevância vêm sendo empreendidas para garantia dos direitos adquiridos e, num esforço conjunto de governos e sociedade, o tema do Consumo Seguro, e sua relação com a Saúde, constitui atualmente um grande desafio e uma discussão importante, na compreensão de que, se a economia está globalizada, nossa ação também precisa ser globalizada, o que tem motivado muitos países a enfrentá-lo de maneira prioritária.

A comunicação eficiente, detecção rápida e a ação coordenada entre países e dentro de cada país, evita a circulação de produtos de consumo inseguros e protege a saúde dos consumidores, mas com a expansão do mercado globalizado, é imprescindível fortalecer e consolidar os sistemas nacionais e regionais de segurança aos produtos.

A RCSS é um instrumento estratégico, potente e eficiente para articular instituições e países nas Américas, compartilhar informações entre os membros dessas organizações e catalisar o desenvolvimento de sistemas sólidos para comunicação de riscos à saúde. É uma estratégia a serviço das autoridades e dos consumidores das Américas. 
O aporte financeiro dos países para as atividades da RCSS é importante, no entanto, mais importante (e fundamental mesmo) é o apoio e acompanhamento políticos dos governos desses países, junto à OEA e OPAS, visando a defesa da continuidade e desenvovlimento da RCSS - para desempenhar forte, intensa e ampla mobilização de todos os países americanos.

No Brasil, diante da suspeita de que o país vive uma epidemia de acidentes de consumo (CBN, 2013), a maior e mais importante prioridade, atualmente, é a produção de esforços interinstitucionais com vistas à implantação do Sistema de Informações de Acidentes de Consumo (SIAC). Por outro lado, em função de sua extensão territorial e diversidade regional, é urgente o desenvolvimento de articulações entre difententes autoridades, fabricantes/empresários e sociedade civil para atuação regional e local, no fortalecimento do movimento Consumo Seguro e Saúde, entendendo Consumo como um novo e importante Determinante da Saúde. Como tal, envolve muitos interesses e diferentes, sendo, portanto um desafio e articulação, também, do campo do Direito Sanitário.

\section{Referências}

ANVISA. I Semana Nacional Consumo Seguro e Saúde, 2013. [on line] Disponível em:

http://portal.anvisa.gov.br/wps/content/anvisa+portal/anvisa/sala+de+imprensa/menu+ +noticias+anos/2013+noticias/semana+discute+relacao+entre+consumo+seguro+e+s aude data da consulta: 8 set. 2013.

ANVISA. Consumo também é questão de saúde. II Seminário Internacional Consumo Seguro e Saúde. 2013. [on line] Disponível em: http://portal.anvisa.gov.br/wps/content/anvisa+portal/anvisa/sala+de+imprensa/menu+ -+noticias+anos/2013+noticias/consumo+tambem+e+questao+de+saude data da consulta: 08 set. 2013.

ANVISA. Rede Consumo Seguro e Saúde. 2010. [on line] Disponível em:

http://portal.anvisa.gov.br/wps/portal/anvisa/anvisa/busca/! ut/p/c5/rVDHlqJQEP2W gCbR4YISHo8QEkSNhyEBkEJgkj4tGZdXdvpmpV555bN2Ax9to2fVZlgi6Nr1hIRYzCbl1qAJA8AePkwCkPEfjof6g4DgWYCGgErdee7hdN6cGiXVJ9Py NmU5M2UZ-DWAelKljFe502w-0i jakatz29dXdUQmHveRNXfny8tCImAdMAN509qqgUazxuhlxD6BKWwxtH4DKU Y3-DgQPyS4yf2tvLcQ uPur sZ yBZhMZucVILTkloDVWdFAGVPUw5AlfUji3n secftWjarVVHiVh3cF b́kU9Kfj102zVKK3wNJ10TK76rMjkha0BRL14IHqvXiSMAqnEJ5zX0bSFiuahTXC7G2356DqYwDiFVtks8PGDeH494ciSNY0mDjINV7aSWDkz8FOH1ZqN0eoi31YXCsG9VILsWKRNMRjn2_gsrjffB9YKHBI 
t59HeLkUZo69tqZHj6SI3eX2jLxZdbEFVALgh9MWdLinl8 Tp-

OCCuOsVseKS8neG8Lf8MZCsS-

6mJF8ZmTv2No1mROyltmQ3TUv4S0ZZA6ZjcXVuPues-

QSfgClJkiYpnMcplsM5isICPVoXaYSzJLzacgjO6tn4MMBSF8807iGE1YIQxleb9LT7a Mf2GR6MgKYb3hYgoU0FGHAzLIcyqOEQqcAVBDMm0ly19_x4zcg8v9VWGOQ7nQk vhgk6laFaCvqr5MqHBdfPtL6LTyd-

HMfng2CMu1Kvuh7zMAwZk1oyg8z9K2ZpXfOF9c0TIYNxXs5xjiw gALxQOx/? $1 \mathrm{dmy \& urile=wcm \% 3apath \% 3a//Anvisa \% 20Portal/Anvisa/Ouvidoria/Publicacao}$ $\% 20$ Ouvidoria/Rede $\% 20$ Consumo $\% 20$ Seguro $\% 20$ e $\% 20$ Saude $\% 20-\% 20$ Voz $\% 20$ da \%200uvidoria\%20-\%20dezembro\%20de\%202010 data da consulta: 8 set. 2013

ANVISA. Audiência sobre Consumo Seguro e Saúde. I Semana de Vigilância Sanitária, 2012. [on line] Disponível em: http://portal.anvisa.gov.br/wps/content/anvisa+portal/anvisa/sala+de+imprensa/menu+ +noticias+anos/2012+noticias/audiencia+sobre+consumo+seguro+e+saude+abre+a+i +semana+de+vigilancia+sanitaria data da consulta: 8 set. 2013

ANVISA. Seminário Internacional discute Consumo Seguro e Saúde I Seminário Internacional Consumo Seguro e Saúde 2012 [on line]. Disponível em: http://portal.anvisa.gov.br/wps/content/anvisa+portal/anvisa/sala+de+imprensa/menu+ +noticias+anos/2012+noticias/seminario+internacional+discute+consumo+seguro+e+ saude data da consulta: 8 set. 2013

BRASIL. Presidência da República. Constituição da República Federativa do Brasil de 1988. $1988 . \quad$ [on line] disponível em: http://www.planalto.gov.br/ccivil 03/constituicao/ConstituicaoCompilado.htm data da consulta: 7 set. 2013.

Determinantes Sociais da Saúde - Portal e Observatório sobre Iniquidades em Saúde. Memória da CMDSS. [Conferência Mundial de Determinantes Sociais da Saúde].

2011. Rio de Janeiro. [on line] Disponível em: http://dssbr.org/site/memoria-dacmdss/. data da consulta: 08 set. 2013.

CBN. Anvisa suspeita que Brasil vive uma epidemia de acidentes de consumo. Entrevista sobre Sistema Nacional de Informações de Acidentes de Consumo 2013. Brasília, CBN. 01/09/2013. [on line] Disponível em: http://cbn.globoradio.globo.com/programas/jornal-da-cbn/2013/09/01/ANVISASUSPEITA-QUE-BRASIL-VIVE-UMA-EPIDEMIA-DE-ACIDENTES-DECONSUMO.htm data da consulta: 8 set. 2013.

ICPHSO. About ICPHSO-International Consumer Product Health and Safety Organization. Acesso em 07/09/2013 e disponí8vel em:

https://www.icphso.org/about.html

IDEC. Idec participa do II Seminário Internacional Consumo Seguro e Saúde. II Seminário Internacional Consumo Seguro e Saúde 2013. Brasília, 20/06/2013. [on line] Disponível em: http://www.idec.org.br/em-acao/em-foco/idec-participa-do-iiseminario-internacional-consumo-seguro-e-saude data da consulta: 8 set. 2013.

INMETRO. Sistema de Monitoramento de Acidentes de Consumo (Sinmac) 2013. Rio de Janeiro, Inmetro, 2013. [on line] Disponível em: http://www.inmetro.gov.br/consumidor/acidente_consumo.asp data da consulta: 7 set. 2013. 
INMETRO. Notícias Consumo Seguro e Saúde. Rio de Janeiro, Inmetro, 2013. [on line] Disponível em: http://www.inmetro.gov.br/busca/busca-google.asp? $\mathrm{cx}=003712908765540286270 \% 3 \mathrm{Abe} 3 \mathrm{fz}$ 4uv0s\&cof=FORID

\%3A9\&ie=iso88591\&q=consumo+seguro+e+saude\&sa=Buscar\&siteurl=www.inmetro. gov.br\%2F\&ref=www.google.com.br\%2Furl\%3Fsa\%3Dt\%26rct\%3Dj\%26q\%3D

\%26esrc\%3Ds\%26frm\%3D1\%26source\%3Dweb\%26cd\%3D1\%26ved

\%3D0CC0QFjAA\%26url\%3Dhttp\%253A\%252F\%252Fwww.inmetro.gov.br\%252F

\%26ei\%3D1Bk1UarVL4KK9QTdiYCwAg\%26usg

\%3DAFQjCNEDJaNgDEeBZqFjz5CJfLMGW03OpQ\%26sig2\%3DiGsX4gnF4mrkBJn6mixUg\&ss=4438j1026732j22 data da consulta: 8 set. 2013.

MINISTÉRIO DA JUSTIÇA. Grupo de Estudos Permanentes de Acidentes de Consumo. Brasília, Ministério da Justiça, 2012. [on line] Disponível em: http://portal.mj.gov.br/main.asp?View=\%7BF84114A3-05C7-41AC-BA85-

936DBE737A1F\%7D\&BrowserType=IE\&LangID=pt-br\&params=itemID\%3D

\%7B8BCC7230-A9E3-4F66-9E95-9F269336A211\%7D\%3B\&UIPartUID=

\%7B2868BA3C-1C72-4347-BE11-A26F70F4CB26\%7D data da consulta: 8 set. 2013

OCDE. Consumer product safety work at the OECD.

Disponí8vel em:

http://www.oecd.org/sti/consumer/consumerproductsafetyworkattheoecd.htm data da consulta: 7 set. 2013 .

OLIVEIRA, NA. Consumo Seguro: um novo determinante social da saúde, um desafio e convite em defesa da Saúde. Blog Direito Sanitário: saúde e cidadania. [on line] disponível em: http://blogs.bvsalud.org/ds/2012/01/11/consumo-seguro-um-novodeterminante-social-da-saude-um-desafio-e-convite-em-defesa-da-saude/ data da consulta: 8 set. 2013.

OLIVEIRA, NA. Congresso de Direito Sanitário no Brasil: oportunidade de integração e fortalecimento de dois importantes movimentos em "REDE". Blog Direito Sanitário: saúde $\quad$ e cidadania. [on line] Disponível em: http://blogs.bvsalud.org/ds/2013/01/14/congresso-de-direito-sanitario-no-brasiloportunidade-de-integracao-e-fortalecimento-de-dois-importantes-movimentos-em\%e2\%80\%9crede\%e2\%80\%9d/ data da consulta: 7 set. 2013.

OLIVEIRA, NA. Além de um observatório, uma rede consumo seguro e saúde. Blog Direito Sanitário: Saude e Cidadania. [on line] Disponível em: http://blogs.bvsalud.org/ds/2010/06/09/alem-de-um-observatorio-uma-rede-consumoseguro-e-saude/ data da consulta: 8 set. 2011.

ORGANIZAÇÃO DOS ESTADOS AMERICANOS (OEA). Brasil relata dificuldade em proteger saúde da população. Audiência Consumo Seguro e Saúde, conjunta na Comissão de Direitos Humans e Comissão de Assintos Sociais do Senado Federal (Brasil), 2013. [on line] Disponível em: http://www.oas.org/es/sla/rcss/Documentos/noticia\%20Anvisa.pdf data da consulta: 8 set. 2013

ORGANIZAÇÃO DOS ESTADOS AMERICANOS OEA. Sobre a RCSS. [Notícias Consumo Seguro e Saúde] 2010. Washington, OEA. 2010. RCSS: http://www.oas.org/pt/sla/rcss/sobre_a_rcss.asp data da consulta: 8 set. 2013. 
ORGANIZAÇÃO DOS ESTADOS AMERICANOS (OEA). Rede Consumo Seguro e Saúde das Américas - Portal de alertas rápidos. [on line] Disponível em: http://www.oas.org/rcss/alerts.aspx?lang=pt data da consulta: 29 dez. 2011.

ORGANIZAÇÃO DOS ESTADOS AMERICANOS (OEA). Rede Consumo Seguro e Saúde das Américas. [on line] Disponível em: http://www.oas.org/pt/sla/rcss/default.asp data da consulta: 7 set. 2013.

ORGANIZAÇÃO MUNDIAL DA SAÚDE. Declaração Política do Rio sobre Determinantes Sociais da Saúde. [on line] Rio de Janeiro: Organização Mundial da Saúde, $\quad$ Brasil, 2011. Disponível em: http://www.who.int/sdhconference/declaration/Rio_political_declaration_portuguese.pd f data da consulta: 7 set. 2013.

O GLOBO Brasil articula redes por consumo seguro. O Globo, Rio de Janeiro, 02/01/2013, Economia, defesa do consumidor. [on line] Disponível em: http://oglobo.globo.com/defesa-do-consumidor/brasil-articula-redes-por-consumoseguro-7176743 data da consulta: 7 set. 2013. 